\title{
Microburst insulin infusion: results of observational studies - carbohydrate metabolism, painful diabetic neuropathy, and hospital/emergency department utilization
}

\begin{abstract}
Background: Pulsed intravenous insulin therapy (PIVIT) has shown equivocal results in studies examining metabolic control. Microburst insulin infusion mimics the periodicity and amplitude of normal pancreatic function more closely than other previous pulsatile insulin therapeutic approaches.

Methods: Data from three observational, retrospective studies are reported: carbohydrate metabolism, painful diabetic neuropathy (PDN), and hospital/emergency department utilization. All studies utilized controlled microbursts of insulin via the Bionica Microdose system in patients with type 1 and type 2diabetes. Indirect calorimetry was used to determine carbohydrate and metabolic rate measures $(\mathrm{n}=311)$. Painful Diabetic Neuropathy (PDN) diagnosis was assessed by the DN4 questionnaire and patients were categorized by neuropathic pain improvement $(\mathrm{n}=412)$. Finally, a two-year study of 1,524 diabetic patients was conducted to compare hospital admissions and emergency department visits to homologous patients.
\end{abstract}

Results: Microburst insulin infusion (MII) treatment increased oxygen consumption and energy expenditure by promoting a greater carbohydrate oxidation rate. Respiratory exchange ratio values increased significantly, reflecting a change toward carbohydrate oxidation, and resting metabolic rate increased $29 \%$. Microburst therapy resulted in complete elimination or reduction of pain in $93 \%$ of PDN patients. Hospital admissions were reduced to $1.65 / 1,000$ / year (versus National Hospital Discharge Survey reported rate of 46.7/1,000/year), and ED visits were reduced to 2.3/1,000/year (versus US Healthcare Statistics rate of 58.4/1,000/year).

Conclusion: Microburst insulin therapy improved underlying carbohydrate metabolism, reduced neuropathic morbidity, and reduced ED visits and hospital admissions in patients with 2 or more diabetic morbidities. Microburst insulin therapy represents a new advance in the treatment of diabetes and the complications of diabetes.

\author{
Volume 4 Issue 4 - 2017 \\ John Elliott,' Nardo Zaias, ${ }^{2}$ Sandra Escova, ${ }^{3}$ \\ Lino Deguzman, ${ }^{4}$ Diane Counce, ${ }^{5}$ Rahul \\ Dixit, ${ }^{6}$ David Capper, ${ }^{7}$ James Novak, ${ }^{8}$ \\ Rosemary Nowins, ${ }^{9}$ Willis Holloway, ${ }^{10}$ \\ Michael Feldman, ${ }^{10}$ Eric Cheung, " Andrew \\ Elliott $M,{ }^{12}$ Allison Cimler, ${ }^{12}$ jill Hopper $M,{ }^{13}$ \\ Ford Gilbert G, ${ }^{14}$ Eric Button $A^{15}$ \\ 'Banner University Medical Center, USA \\ ${ }^{2}$ Greater Miami Skin and Laser Center, USA \\ ${ }^{3}$ Herbert Wertheim College of Medicine, USA \\ ${ }^{4}$ Loma Linda University Newport Beach, USA \\ ${ }^{5}$ Trina Health of Birmingham, USA \\ ${ }^{6}$ Rina Health of West Los Angeles, USA \\ ${ }^{7}$ Advanced Metabolic Care Associates, USA \\ ${ }^{8}$ Trina Health of San Diego, USA \\ ${ }^{9}$ Trina Health of Las Vegas, USA \\ ${ }^{10}$ Trina Health of Oklahoma, USA \\ "Trina Health of Foley, USA \\ ${ }^{12}$ Trina Health of Arizona, USA \\ ${ }^{13}$ Trina Health of Dallas, USA \\ ${ }^{14}$ Trina Health, USA \\ ${ }^{15}$ NeoMark Ventures, USA
}

Correspondence: Ford Gilbert G, Founder and CEO of Trina Health, USA, Email kevin.shippy@gmail.com

Received: June 16, 2017 | Published: July 14, 2017

\section{Background}

Recent research has demonstrated the burst insulin secretion of pancreatic beta cells in response to a carbohydrate load. A convincing body of evidence indicates that insulin is secreted in synchronized bursts from the pancreas into the hepatic portal vein..$^{1-3}$ Multiple studies in humans and animals have described the resulting oscillatory nature of systemic levels of blood glucose and insulin. ${ }^{4-7}$ Moreover, the loss of pulsatile pancreatic activity and B-cell dysfunction may not only be initiating adverse events, but also contribute to the development of hepatic insulin resistance and progression of type 2 diabetes. ${ }^{8}$

Providing insulin in a burst fashion using a device delivering small bursts of exogenous insulin would be expected to affect insulin target tissues more effectively by more closely mimicking the secretion of insulin observed in normal individuals. In previous studies, pulsed intravenous insulin delivery has shown encouraging, yet mixed results in lowering blood glucose levels compared to equal doses of continuously infused insulin..$^{9,10}$ Compared to standard therapy, burst therapy has shown promising, yet equivocal results in studies examining metabolic control, end-organ damage, and restoration of normal pulsatile pancreatic function in type 2 diabetes. ${ }^{11-13}$ Perhaps the most encouraging study on the effects of pulsed therapy on endorgan damage showed a significant preservation of renal function by pulsatile insulin infusion. ${ }^{14}$

These previous studies used earlier variations of what has been called pulsatile intravenous insulin therapy (PIVIT), which does not effectively mimic normal human insulin bursts. PIVIT, also referred to as outpatient intravenous insulin therapy (OIVIT), chronic intermittent intravenous insulin infusion (CIIIT), hepatic activation therapy (HAT), metabolic activation therapy (MAT) or the Harvard Protocol, does not replicate the periodicity and amplitude of normal pancreatic function, and was designed to deliver insulin in a series of increasing insulin peaks, producing successively increasing free insulin with limited clinical effectiveness.

In contrast, "microburst insulin infusion" (MII) via the Bionica Microdose Pump achieves the microburst of insulin causing oscillations with an amplitude of normal insulin secretion without an ever-increasing baseline of insulin - thus more closely mimicking natural burst insulin secretion. 
In this report, we highlight data, which underscore the effectiveness of microburst insulin infusion in three observational, retrospective studies. These studies specifically focus on the effects of MII on metabolic rate measures, the potential attenuation and reversal of pain in patients with diabetic neuropathy, and on outcome measures as determined by hospital admissions and emergency department utilization.

\section{Methods}

All studies used MII which consisted of 3 one-hour insulin treatments, during which infused, controlled microbursts of IV insulin via the Bionica Microdose Pump, are given to match a preset measured oral ingestion of glucose equaling the average caloric need for that patient's weight during the period of treatment. A rest period of up to 30minutes is given between the one-hour microburst infusions. The treatment was calculated and delivered according to the Bionica IV infusion device Supervisor's Guide and Trina Health protocols. ${ }^{15}$ Patients in all 3 studies continued to receive their regular regimen of hypoglycemic medication either oral or insulin.

Restoration of more normal resting metabolism of carbohydrates was assessed using the Vacumed Vista-MX2 Metabolic Measurement System, a breath-by-breath mixing chamber measurement system of the volume of carbon dioxide produced at rest. The resting carbohydrate utilization measurements were taken 3 times per treatment day. The first measurement is before initiation of MII treatment, then midway in the treatment and the third measurement at the finish of the treatment regimen. Changes in $\mathrm{VCO}_{2}$ are diagnostic, and are used to verify patient resting metabolism responses to the Trina Health protocol, and to determine the necessary treatment frequency (from once per week to once per three weeks or longer). Respiratory Quotients and VCO2 measurements were not used to guide and adjust treatment, but rather to determine the necessary frequency of treatment to maintain proper carbohydrate metabolism.

The individual treatment protocol for each patient continues until the patient's metabolic status is maintained over a personalized period of time as shown by the ability to quickly increase carbohydrate utilization in the presence of an oral glycemic load during the first hour of treatment. The first 2 treatments are given within 1-3days of each other, and thereafter treatment is not continuous or pre-set as to the number of days between treatments.

\section{Carbohydrate metabolism study}

Indirect calorimetry is the primary method for metabolic rate measurement and involves measurement of oxygen consumption $\left(\mathrm{VO}_{2}\right)$ and carbon dioxide production $\left(\mathrm{VCO}_{2}\right) \cdot{ }^{16}$ The relation between these gases and metabolic rate is defined by the respiratory exchange ratio (RER), Resting Metabolic Rate (RMR), and other measures. RER, which is a measurement of carbon dioxide produced per unit of oxygen $\left(\mathrm{VCO}_{2} / \mathrm{VO}_{2}\right)$, varies with type of substrate (carbohydrate, fat, protein). An increased RER value is indicative of carbohydrate oxidation being favored over the oxidation of lipids for energy production. RMR is the measurement of energy required to maintain basic body functions while in a state of rest and accounts for $65 \%$ to $75 \%$ of total energy expenditure. The aim of this study was to examine the effects of MII therapy on metabolic rate measures as determined by indirect calorimetry.

Study metodology: This retrospective analysis included 311 patients treated at 12 centers, representing 7,583 individual microburst insulin treatments. Baseline and post-treatment $\mathrm{VCO}_{2}$ and $\mathrm{VO}_{2}$ metabolic measurement readings were taken for each individual treatment and comprehensive metabolic profiles were calculated. RER was determined by the respiratory exchange ratio (VCO2/VO2). Resting metabolic rate (RMR) was determined by the Weir equation. ${ }^{17}$ The Jeukendrup \& Wallis equation was used to calculate carbohydrate (CHO) oxidation..$^{18}$ Carbohydrate measures include \% $\mathrm{CHO}$ oxidation of the total kcals expended, $\mathrm{CHO}$ kcals oxidized per liter of oxygen consumed, and $\mathrm{CHO}$ kcals oxidized per minute. Post-hoc analysis was performed using an orthogonal pair-wise contrasting process adjusted for within subject individual variances determined by the original repeated measures design.

Results: Oxygen consumption significantly changed over the three measurement periods (Table 1). Oxygen consumption increased from baseline to measurement periods 2 and 3 by $1.9 \%(\mathrm{p}=0.05)$ and $4.4 \%(p<0.0001)$, respectively. Comparing oxygen consumption between measurements 2 and 3 showed that the period 3 measurement was greater by $2.5 \%(\mathrm{p}=0.015)$. $\mathrm{VCO}_{2}$ showed similar trends, but to a greater degree. Changes in $\mathrm{VCO}_{2}$ production comparing baseline measurement periods 2 and 3 were $2.6 \%(p=0.03)$ and $9.7 \%(p=0.001)$, respectively. Comparing measurement periods 2 and $3, \mathrm{VCO}_{2}$ production increased $7.1 \%(\mathrm{p}<0.0001)$. The RER values at baseline and across the other two measurement periods indicate that from baseline to period 2 , the respective changes in oxygen consumption and the production of $\mathrm{VCO}_{2}$ responded similarly. RER did not significantly change initially (Baseline RER $=0.91 \pm 0.11$; Period 2 $\mathrm{RER}=0.92 \pm 0.13, \mathrm{p}=\mathrm{NS}$ ). However, due to a greater relative increase in $\mathrm{VCO}_{2}$ production versus $\mathrm{VO}_{2}$ consumption in the final measurements, period 3's RER significantly increased to both baseline and the period 2 measurement $(p<0.0001)$. As a result, both the amount of $\mathrm{CHO}$ oxidized in kcals per minute and the $\mathrm{CHO}$ percentage of the total resting energy expenditure was significantly greater following both microburst insulin infusion treatment periods. As a result of multiple microburst insulin infusions, carbohydrate oxidation in kcals per liter of $\mathrm{O}_{2}$ increased for each measurement period (Figure 1). RMR values increased significantly from baseline to period $3(p<0.0001)$ and also increased significantly from period 2 to period $3(\mathrm{p}=0.015)$. Baseline RMR values from the first visit to last visit over a period of 3 months were also compared in 27 patients in one clinic (Figure 2). Mean baseline RMR values increased from 1,475 to $1,904(\mathrm{p}<0.0001)$, an increase of $29 \%$.

Study conclusion: MII treatment significantly increased oxygen consumption and energy expenditure by promoting a greater carbohydrate oxidation rate both in absolute (CHO oxidation in kcals per liter of $\mathrm{O} 2$ consumed and $\mathrm{CHO}$ oxidation in kcals per minute) and relative terms (percentage of $\mathrm{CHO}$ kcals expended relative the treatment groups total kcals expended per minute at rest). The increase in the respiratory exchange ratio soon after the introduction of microburst insulin therapy is to be expected, given the well-known actions of insulin on promoting glucose uptake and carbohydrate oxidation while reducing lipolysis and lipid oxidation, ${ }^{19}$ and these effects are reduced by insulin resistance, yet are still present in patients with type 2 diabetes..$^{20} \mathrm{~A}$ raised RER shows that insulin is favoring carbohydrate oxidation rather than lipids for energy production. Previous investigators have reported decreases in resting metabolic rate over the short-term (2-8days) in patients treated with insulin. ${ }^{21-23}$ It is thought that the immediate decreases in RMR may be due to the short-term underlying effects of insulin on the reduction of energyconsuming processes such as proteolysis and gluconeogenesis. ${ }^{24}$ We also report an immediate decrease in RMR in the second period following MII, consistent with previous findings. However, there 
was a subsequent increase in the last period, and a very significant increase of $29 \%$ in RMR values baseline-to-baseline over 3months. These results suggest that MII has a dramatic effect on carbohydrate metabolism, overcoming the reduction of resting metabolic rate seen with conventional insulin treatments. This is of particular importance as the inability to properly metabolize carbohydrates represents a core dysfunction in diabetes. By preferentially converting energy production to carbohydrate metabolism, relative to lipids, diabetic patients avoid the consequences of elevated free fatty acids, which trigger a cascade of inflammatory processes.

Table I Metabolic Effects of Multi-Micro Insulin Burst $\mathrm{OnVO}_{2}, \mathrm{VCO}_{2}$, and Substrate Oxidation

\begin{tabular}{|c|c|c|c|c|c|c|c|}
\hline Variable & $\begin{array}{l}\text { Baseline } \\
\text { mean }(n \\
=311)\end{array}$ & $95 \% \mathrm{Cl}$ & $\begin{array}{l}\text { Period } \\
2 \text { Mean } \\
(n=73)\end{array}$ & $95 \% \mathrm{Cl}$ & $\begin{array}{l}\text { Period } 3 \\
\text { mean } \\
(n=3 I I)\end{array}$ & $95 \% \mathrm{Cl}$ & Statistical Comparisons \\
\hline VCO2 (liters • min-I) & 246.9 & $239.3-254.5$ & 247.3 & $230.1-264.5$ & 270.5 & $262.5-278.5$ & $\begin{array}{l}\text { Baseline vs Period } 2: p=0.0303 \\
\text { Baseline vs Period } 3: p<0.000 \text { I Period } \\
2 \text { vs Period } 3: p<0.0001\end{array}$ \\
\hline RER (VCO2/VO2) & 0.91 & $0.90-0.93$ & 0.93 & $0.90-0.96$ & 0.96 & $0.95-0.97$ & $\begin{array}{l}\text { Baseline vs Period } 2: p=\text { NS Baseline } \\
\text { vs Period } 3: p<0.000 \text { I Period } 2 \text { vs } \\
\text { Period 3: } p<0.000 \text { I }\end{array}$ \\
\hline $\mathrm{CHO}$ oxidized (\%) & 66.1 & $63.2-68.9$ & 72.3 & $67.1-77.6$ & 78 & $75.8-80.3$ & $\begin{array}{l}\text { Baseline vs Period } 2: p=0.0450 \\
\text { Baseline vs Period } 3: p<0.000 \text { I Period } \\
2 \text { vs Period } 3: p=0.001\end{array}$ \\
\hline $\begin{array}{l}\mathrm{CHO} \text { oxidized (kcals/ } \\
\text { min-I) }\end{array}$ & $0.87 I$ & $0.827-0.916$ & 0.962 & $0.867-1.057$ & 1.095 & $1.05 \mathrm{I}-\mathrm{I} .140$ & $\begin{array}{l}\text { Baseline vs Period } 2: p=0.0430 \\
\text { Baseline vs Period } 3: p<0.000 \text { I Period } \\
2 \text { vs Period } 3: p<0.000 \text { I }\end{array}$ \\
\hline $\begin{array}{l}\text { Resting metabolic } \\
\text { rate (kcals) }\end{array}$ & 1,916 & $|, 86|-\mid, 972$ & I,882 & I,774-I,990 & 2,021 & I,966-2,077 & $\begin{array}{l}\text { Baseline vs Period } 2: p=0.3672 \\
\text { Baseline vs Period } 3: p<0.0001 \text { Period } \\
2 \text { vs Period } 3: p=0.015\end{array}$ \\
\hline
\end{tabular}

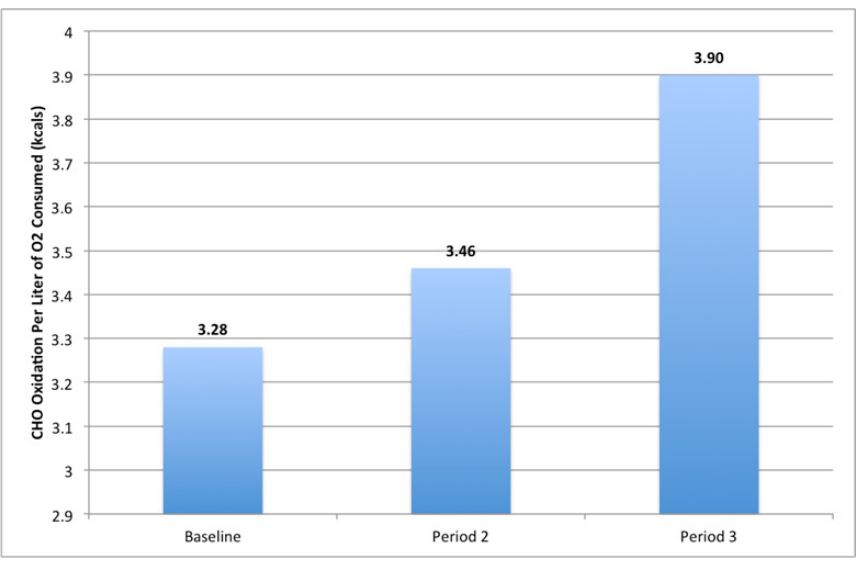

Figure I Effects of Microburst Insulin Infusion on Mean $\mathrm{CHO}$ Oxidation Over Time. Period $2>$ Baseline $=5.5 \% \quad(p=0.05)$, Period $3>$ Baseline $=18.9 \%$ $(p<0.000 \mathrm{I})$, Period $3>$ Period $2=12.7 \%(p<0.000 \mathrm{I})$

\section{Painful diabetic neuropathy study}

Diabetic neuropathy is one of the most common microvascular complications of diabetes. Painful diabetic neuropathy (PDN) is reported in $16-34 \%$ of patients with diabetes..$^{25}$ The symptoms of PDN can be debilitating and can cause sleep disturbances, anxiety, and interfere with physical functioning. ${ }^{26}$ The lack of normal sensation leads to injuries and eventually to amputation in many patients. PDN represents an ongoing therapeutic challenge and no single treatment exists to prevent or reverse neuropathic changes or to provide significant pain relief. Three classes of drugs are commonly used to treat diabetic neuropathy: tricyclic antidepressants, anticonvulsants, and selective serotonin-reuptake inhibitors. These compounds, however, have limited effectiveness, necessitating the need for additional pain management approaches. ${ }^{27}$ This retrospective study analyzed the effects of MII on pain associated with diabetic neuropathy.

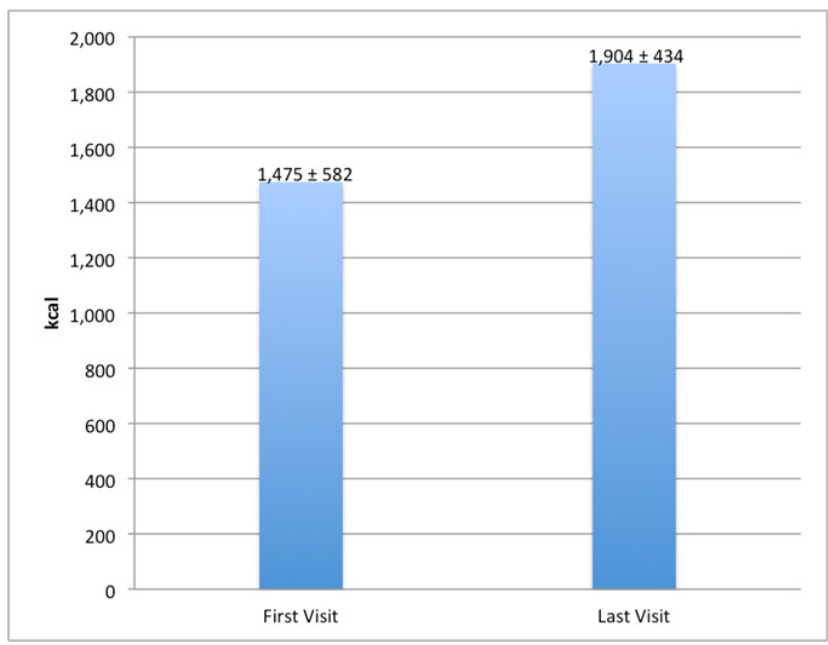

Figure 2 Comparison of Mean Resting Metabolic Rate (Baseline to Baseline). Means \pm SD Last Visit $>$ First Visit $=29.1 \%(p<0.0001)$

Study methodology: The study included 412 diabetic patients (81.1\% Type $2 \mathrm{DM}$, and $19.9 \%$ Type $1 \mathrm{DM}$ ), there were 273 males and 204 females with a mean age of 58.3years. These patients were being treated with MII therapy as previously described. Of these 412 
patents, 299 patients $(72.6 \%)$ were diagnosed as having significant Painful Diabetic Neuropathy (PDN). Patients with PDN were then treated with microburst insulin therapy once per week over a period of 3 months. The diagnosis of PDN was based on clinical findings: type of pain (burning discomfort, electric shock-like sensation, aching coldness in the lower limbs); time of occurrence (most bothersome at rest and at night); and abnormal or missing sensation (such as tingling or numbness) as determined by the DN4 questionnaire. ${ }^{28}$ No neurological treatment other than MII treatment was provided, and no analgesic medications were used. Patients were then categorized based on neuropathic improvement (no improvement, significant improvement, and complete resolution), and percentages were compared (t-test, comparing percentages).

Results: After 3months, 142 patients (47.5\%) completely resolved all neuropathic symptoms, 136patients (45.5\%) reported significant improvement without complete resolution, and 21 patients $(7.0 \%)$ reported no improvement (Table 2). There were significant differences (t-test) when comparing the percentages of both significantly improved and completely resolved patients to patients that were not improved $(\mathrm{p}<.0001)$.

Table 2 Neuropathic Improvement - One-sample t-test between percentages $(n=299)$

\begin{tabular}{ll}
\hline No improvement & Significant improvement \\
\hline $\mathrm{N}=2 \mathrm{I}(7 \%)$ & $\mathrm{N}=136(45.5 \%)$ \\
& $\mathrm{P}$-value $<.000 \mathrm{I}$ (versus no improvement) \\
\hline
\end{tabular}

Study conclusion: Various symptomatic treatments have been proposed to manage neuropathic pain, but few have been found to be effective, with only three medications currently FDA approved for PDN. It is noteworthy that MII showed complete elimination or significant reduction of pain in $93 \%$ of PDN patients after 3 months treatment-with complete resolution of pain in $47 \%$ of PDN patients. Such significant reductions in pain may, in fact, be due to the mimicking of natural pancreatic function and restoration of carbohydrate metabolism by MII, but the exact mechanism requires further study. Given the lack of effective therapies for PDN and the encouraging findings reported in this study, additional studies on the effect of MII in PDN patients are warranted.

\section{Hospital and emergency room utilization study}

A retrospective analysis was conducted to determine if MII has a beneficial effect on patients with diabetes as determined by hospital and emergency room utilization when compared to matched National Hospital Discharge Survey and US Agency for Healthcare Statistics for homologous patients.

Methodology: This was a two-year retrospective study conducted at 14 centers involving 1,524 Type 1and Type 2 DM patients, with two or more secondary complications of diabetes (Table 3), and included patients with histories of multiple hospitalizations and emergency department visits prior to initiation of MII treatment. All study patients were treated with the MII protocol as described previously in addition to their regular hypoglycemic therapy.

Results: During the two-year study period, there were a total of 5 admissions to the hospital for treatment (Table 4). The expected hospitalization rate for patients with diabetes and two or more morbidities as determined by the National Hospital Discharge Survey (NHDS) over a two-year study period is 96 per 1000 diabetic patients
(47 per 1000 diabetic patients per year). ${ }^{29}$ There were a total of 7 visits to the emergency department (ED) for the same 1,524 patients over the 2year study period (Table 5). The expected number of emergency department visits over two years is 116 per 1,000 diabetic patients (58 per 1,000 diabetic patients per year) as determined by the US Agency for Healthcare Statistics (USAHS) ${ }^{30}$ When comparing the rates of expected hospitalization and emergency department visits versus the actual number of visits for patients treated with MII treatment, there was a significant difference between the populations $(\mathrm{p}<0.0001)$.

Study conclusion: MII reduced hospitalization in a large group of diabetics with 2 or more serious co-morbidities to $1.65 / 1,000 /$ year (versus NHDS estimated rate of 46.7/1,000/year) and emergency department visits to 2.3/1,000/year (versus US for Healthcare Statistics estimated rate of 58.4/1,000/year). This represents a significant reduction in hospital admissions and emergency department visits compared to normal rates-presumably generating substantial savings for the health care system.

Table 3 Comorbidities from Diabetes among study patients

Diabetic Nephropathy, stages I - 4 (with and without dialysis)

Neuropathy, hands, feet, and other sites

Cardiomyopathy, CVD

Retinopathy, proliferative and other

Vascular dementia, (often associated with un-healing wounds)

Hypertension, (medication reductions)

Un-healing wounds, (with and without osteomyelitis)

Skin pigmentation

Sleeplessness, Restless Leg Syndrome

Functional energy loss

Fibromyalgia

Hyperlipidemia

Hypoglycemia unawareness (TIDM)

Black toes and feet (avoided amputations)

Gastroparesis

Insulin resistance

Myasthenia gravis (MG) significant improvement

Balance disorders

Gait disorders, non-orthopedic

Erectile dysfunction

Cramping

Depression (related)

Depression by family/significant others

Table 4 Hospitalization Visits

Comparison of two rates (Adjusted for 2Years) Study Visits NHDS Rate

Numerator (e.g. number or events counted) $5 \quad 94$

Denominator (e.g. total personyears)
94

1524

1000

Citation: Elliott J, Zaias N, Escovar S, et al. Microburst insulin infusion: results of observational studies - carbohydrate metabolism, painful diabetic neuropathy, and hospital/emergency department utilization.J Diabetes Metab Disord Control. 2017;4(4):I I6-121. DOI:10.15406/jdmdc.2017.04.00II8 
Table continued.

Comparison of two rates (Adjusted for 2Years)

\begin{tabular}{ll}
\hline & Study Visits NHDS Rate \\
\cline { 2 - 2 } Study incidence rate & $0.00328 \mathrm{I}$ \\
95\% Confidence Interval & 0.001065 to 0.007656 \\
CDC incidence rate & 0.047 \\
95\% Confidence Interval & 0.03453 to 0.0625 \\
Incidence rate difference & -0.04372 \\
95\% Confidence Interval & -0.05517 to -0.03227 \\
P-value & $\mathrm{P}<0.000 \mathrm{I}$ \\
\hline
\end{tabular}

Table 5 Emergency Department Visits

\begin{tabular}{|c|c|c|}
\hline \multicolumn{3}{|c|}{ Comparison of two rates (Adjusted For 2Years) } \\
\hline & $\begin{array}{l}\text { Study } \\
\text { visits }\end{array}$ & $\begin{array}{l}\text { USAHS } \\
\text { rate }\end{array}$ \\
\hline Numerator (e.g. number or events counted) & 7 & 116 \\
\hline Denominator (e.g. total person-years) & 1524 & 1000 \\
\hline Study incidence rate & \multicolumn{2}{|c|}{0.004593} \\
\hline 95\% Confidence Interval & \multicolumn{2}{|c|}{$\begin{array}{l}0.001847 \text { to } \\
0.009464\end{array}$} \\
\hline USAHS incidence rate & \multicolumn{2}{|l|}{0.058} \\
\hline $95 \%$ Confidence Interval & \multicolumn{2}{|c|}{$\begin{array}{l}0.04404 \text { to } \\
0.07498\end{array}$} \\
\hline Incidence rate difference & \multicolumn{2}{|c|}{-0.05341} \\
\hline 95\% Confidence Interval & \multicolumn{2}{|c|}{$\begin{array}{l}-0.06621 \text { to } \\
-0.04061\end{array}$} \\
\hline P-value & \multicolumn{2}{|c|}{$P<0.0001$} \\
\hline
\end{tabular}

\section{Discussion}

Pulsatile intravenous insulin therapy (PIVIT), referred to by several different names, attempted to provide insulin in a novel way, a pulsatile pattern of insulin, which met with encouraging but limited success..$^{13,31-32}$ This therapy pre-dated widespread medical knowledge of how a normal pancreas secrets insulin in microbursts every 4-6minutes. ${ }^{33}$ An alternative approach, microburst insulin infusion (MII), has been developed that is more similar to physiologic insulin release, without an ever-increasing baseline of insulin that was inherent with previous approaches (PIVIT). MII differs from PIVIT and mimics both the periodicity and amplitude of normal pancreatic insulin release more closely. This novel therapy is used as a supplement to regular hypoglycemic therapy and provides advantages in managing patients with both type 1 and type 2diabetes and associated secondary complications. In this study, we report the results of three observational studies that utilize MII in normal, clinical settings-the first focusing on metabolic rate measures related to carbohydrate metabolism, the second on neuropathy, a common microvascular complication, and the third on hospital admission/ emergency department outcome data. Significant improvements in oxygen consumption and carbon dioxide production were shown consistently over 7,583 treatments in multiple clinics, reflecting underlying improvement in carbohydrate metabolism. As far as we know, this is also the first report of an insulin treatment regimen that actually increases the resting metabolic rate (RMR) over a longer term in patients with diabetes. The increase in RMR of $29 \%$ over a period of three months is most notable. The improvements in carbohydrate metabolism by MII treatment have several implications related to systemic inflammation. The conversion of energy production to carbohydrate substrates relative to lipids minimizes the production of free fatty acids which trigger the inflammation cascade. ${ }^{34}$ Inflammation affects insulin signaling and increases beta-cell death, and markers of inflammation such as elevated interleukin 6 (IL-6) and C-reactive protein (CRP) levels (Reference-Whitehall). Thus, MII may have considerable impact on the course of chronic inflammation in patients with diabetes. Future studies will further investigate the link between MII and inflammation, as well as the effects on markers of inflammation. Compared to traditional pharmaceutical treatment approaches for painful diabetic neuropathy, MII had notable results - with either complete elimination or significant reduction in pain in $93 \%$ of patients. These results are important as painful diabetic neuropathy (PDN) is common and is associated with significant impairment in the quality of life in patients with diabetes. Given the very few effective treatment options for patients with PDN, future studies are planned to both confirm these clinical results and investigate underlying mechanisms.

Assessment of the population health care impact of MII was demonstrated by the outcome data generated over two years, assessing hospital admissions and emergency department visits. Both these measures showed statistically significant reductions when compared to standard benchmarks, over the entire spectrum of secondary diabetes complications. Such reductions would presumably translate into substantial savings for the health care system at multiple levels. A limitation of these studies was the lack of double-blinded controls. This was deemed impractical due to the treatment duration of four hours, where glucose is given to diabetic patients with significant comorbidities. Hence, we used well-documented historical data from studies for comparative purposes. These retrospective studies we report here were typically conducted over a wide range of clinical settings and geographical locations.

\section{Conclusion}

In summary, conventional insulin therapy has achieved only partial success in the treatment of diabetes and prevention of chronic complications. Disappointing insulin efficacy in the treatment of diabetes may be less due to the quality of exogenous insulin than due to the possibility of physiologic "inadequacy" of its method of administration. Although insulin is secreted normally from pancreatic beta cells in a pulsatile manner, the current methods of therapeutic insulin administration are not pulsatile. Investigators have attempted to develop different methodologies to mimic normal pulsatile insulin delivery with limited success.

Microburst insulin therapy represents a new advance in the treatment of diabetes, due to its ability to closely replicate normal pancreatic function. Taken together, the rather dramatic reductions in neuropathic complications and hospital/emergency department visits, as well as the significant improvements in carbohydrate metabolism in these observational studies, may be due to the more precise mimicking of naturally pulsed insulin.

\section{Acknowledgements}

None.

\section{Conflict of interest}

Author declares that there is no conflict of interest. 


\section{References}

1. Cook DL. Isolated islets of Langerhans have slow isolations of electrica activity. Metabolism. 1983;32:681-685.

2. Bergsten P, Grapengiesser E, Gylfe E, et al. Synchronous oscillations of cytoplasmic $\mathrm{Ca}^{2}+$ and insulin release in glucose-stimulated pancreatic islets. J Biol Chem. 1994;269(12):8749-8753.

3. Zhang M, Goforth $\mathrm{P}$, Bertram $\mathrm{R}$, et al. The $\mathrm{Ca}^{2}+$ dynamics of isolated mouse beta-cells and islets: implications for mathematical models. Biophys J. 2003;84(5):2852-2870.

4. Lang DA, Matthews DR, Peto J, Turner RC (1979) Cyclic oscillations of basal plasma glucose and insulin concentrations in human beings. $N$ Engl J Med. 1979;301(19):1023-1027.

5. Matveyenko AV, Veldhuis JD, Butler PC. Measurement of pulsatile insulin secretion in the rat: direct sampling from the hepatic portal vein Am J Physiol Endocrinol Metab. 2008;295(3):E569-E574.

6. Porksen N, Nyholm B, Veldhuis JD, et al. In humans at least $75 \%$ of insulin secretion arises from punctuated insulin secretory bursts. $\mathrm{Am} \mathrm{J}$ Physiol. 1997;273(5 Pt 1):E908-E914.

7. Song SH, McIntyre SS, Shah H, et al. Direct measurement of pulsatile insulin from the portal vein in human subjects. J Clin Endocrinol Metab. 2000;85(12):4491-4499.

8. Wahren J, Kallas A. Loss of pulsatile insulin secretion: A factor in the pathogenesis of type 2 diabetes. Diabetes. 2012;61(9):2228-2229.

9. Matthews DR, Naylor BA, Jones RG, et al. Pulsatile insulin has greater hypoglycemic effect than continuous delivery. Diabetes. 1983;32(7):617-621.

10. Komjati M, Bratusch-Marrain P, Waldhausl W. Superior efficacy of pulsatile versus continuous hormone exposure on hepatic glucose production in vitro. Endocrinology. 1986;118(1):312-319.

11. Aoki TT, Grecu EO, Arcangeli MA, et al. Chronic intermittent intravenous insulin therapy: a new frontier in diabetes therapy. Diabetes Technol Ther. 2001;3(1):111-123.

12. Dailey GE, Boden GH, Creech RH, et al. Effects of pulsatile intravenous insulin therapy on the progression of diabetic nephropathy. Metabolism. 2000;49(11):1491-1495.

13. Mirbolooki MR, Taylor GE, Knutzen VK, et al. Pulsatile intravenous insulin therapy: the best practice to reverse diabetic complications? Med Hypotheses. 2009;73:363-369.

14. Weinrauch LA, Sun J, Gleason RE, et al. Pulsatile intermitten intravenous insulin therapy for attenuation of retinopathy in type 1 diabetes mellitus. Metabolism. 2010;59(10):1429-1434.

15. Supervisor's Guide and Trina Health protocols (need specific reference)

16. Haugen HA, Chan LN, Li F. Indirect Calorimetry: A Practical Guide for Clinicians. Nutr Clin Pract. 2007;22(4):377-388.

17. Weir JB. New methods for calculating metabolic rate with special reference to protein metabolism. J Physiol. 1949;109(1-2):1-9.
18. Jeukendrup AE, Wallis GA. Measurement of substrate oxidation during exercise by means of gas exchange measurements. Int J Sports Med. 2005;26(Suppl 1):S28-S37

19. Laville M, Rigalleau V, Riou JP, et al. Respective role of plasma nonesterified fatty acid oxidation and total lipid oxidation in lipidinduced insulin resistance. Metabolism. 1995;44(5):639-644.

20. Taskinen MR, Bogardus C, Kennedy A, et al. Multiple disturbances of free fatty acid metabolism in noninsulin-dependent diabetes. Effect of oral hypoglycaemic therapy. J Clin Invest. 1985;76(2):637-644.

21. Buscemia S, Donatelli M, Grosso G, et al. Resting energy expenditure in type 2 diabetic patients and the effect of insulin bolus. Diabetes Res Clin Pract. 2014;106(3):605-610.

22. Gonzales C, Fagour C, Maury E, et al. Early changes in respiratory quotient and resting energy expenditure predict later weight changes in patients treated for poorly controlled type 2 diabetes. Diabetes Metab. 2014;40(4):299-304.

23. Fagour C, Gonzalez C, Suberville C, et al. Early decrease in resting energy expenditure with bedtime insulin therapy. Diabetes Metab. 2009;35(4):332-335.

24. Nair KS, Garrow JS, Ford C, et al. Effect of poor diabetic control and obesity on whole body protein metabolism in man. Diabetologia. 1983;25(5):400-403.

25. Abbott C, Malik R, Van Ross E, Kulkarni J, et al. Prevalence and characteristics of painful diabetic neuropathy in a large community-based diabetic population in the U.K. Diabetes Care. 2011;34(10):2220-2224.

26. Galer BS, Gianas A, Jensen MP. Painful diabetic neuropathy: epidemiology, pain description, and quality of life. Diabetes Res Clin Pract. 2000;47(2):123-128.

27. Javed S, Petropoulos N, Alam U, et al. Treatment of painful diabetic neuropathy. Ther Adv Chronic Dis. 2015;6(1):15-28.

28. Spallone V, Morganti R, D'Amato C, et al. Validation of DN4 as a screening tool for neuropathic pain in painful diabetic polyneuropathy. Diabet Med. 2012;29(5):578-585.

29. Hall JM, DeFrances J, Williams SN, et al. National Hospital Discharge Survey: 2007 Summary. Natl Health Stat Report. 2010;29:1-24.

30. Washington RE, Andrews RM, Mutter R. Emergency Department Visits for Adults with Diabetes, 2010. Healthcare Cost and Utilization Project (HCUP) - Statistical Brief 167. 2013

31. Hellman B (2009) Pulsatility of insulin release - a clinical important phenomenon. Ups J Med Sci. 2009;114(4):193-205.

32. Satin LS, Butler PC, Ha J, et al. Pulsatile insulin secretion, impaired glucose tolerance and type 2 diabetes. Mol Aspects Med. 2015;42:61-77.

33. Porksen N, Munn SR, Steers JL, et al. Effects of glucose ingestion versus infusion on pulsatile insulin secretion. Diabetes. 1996;45(10):1317-1323.

34. Marmot MG, Davey Smith G, Stansfield S, et al. Health inequalities among British civil servants: the Whitehall II study. Lancet. 1991;337(8754):1387-1393. 